\title{
A Study to Evaluate the Relevance and Validity of Triple Assessment in Diagnosing Carcinoma Breast
}

\author{
Nirmal Kumar Sinha ${ }^{1}$, Nishi Kumari ${ }^{2}$ \\ ${ }^{1}$ Department of General Surgery, NMCH, Patna, Bihar, India. \\ ${ }^{2}$ Department of General Surgery, NMCH, Patna, Bihar, India.
}

\section{ABSTRACT}

\section{BACKGROUND}

Breast cancer is the second leading cause of cancer related deaths. Breast cancer survival is strongly determined based on the stage of the cancer after diagnosis. This demonstrates the benefits of early detection of breast cancer in keeping survival rates of patients higher. Triple Test that comprises clinical examination, radiological assessment (USG/Mammography) and FNAC is considered as the gold standard in diagnosing carcinoma breast. This study aimed at diagnosing carcinoma breast in patients with the help of triple assessment and studying its relevance and validity.

\section{METHODS}

Women who presented with a lump in breast to surgery OPD in the department of general surgery, NMCH, Patna were included in the study. This is a prospective study undertaken from December 2017 to November 2019. After taking informed consent from the study participants, they were thoroughly examined, and detailed history was taken. Subsequently, they were subjected to other components of triple test, so that a definitive diagnosis could be formed. Cases of breast abscess and those with fungating or ulcerative mass were excluded from the study.

\section{RESULTS}

Clinical examination had a sensitivity of $90 \%$ and specificity of $86.66 \%$. FNAC had a sensitivity of $95 \%$ and specificity of $96.66 \%$. Radiological assessment when used alone, yielded a sensitivity of $90 \%$ and specificity of $96.66 \%$. The sensitivity and specificity of all the modalities used in triple assessment when combined together was $100 \%$ and $96.66 \%$ respectively. The positive predictive value was $95.23 \%$ and negative predictive value was $100 \%$. The $\mathrm{p}$ value was highly significant $(<0.0001)$.

\section{CONCLUSIONS}

Triple assessment is an important diagnostic tool in the assessment of carcinoma breast and is better than either of the modalities used alone.

\section{KEY WORDS}

Triple Assessment, Carcinoma Breast, Sensitivity, Specificity
Corresponding Author:

Dr. Nishi Kumari,

Flat-203/A, Saryug Tower,

Bhootnath Road,

Kankarbagh, Patna-800026,

Bihar, India.

E-mail: nishi.nmch@gmail.com

DOI: $10.14260 / j e m d s / 2020 / 301$

Financial or Other Competing Interests: None.

How to Cite This Article:

Sinha NK, Kumari N. A study to evaluate the relevance and validity of triple assessment in diagnosing carcinoma breast. J. Evolution Med. Dent. Sci. 2020;9(16):1386-1389, DOI: 10.14260/jemds/2020/301

Submission 14-02-2020, Peer Review 03-04-2020, Acceptance 10-04-2020, Published 20-04-2020.

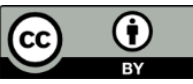




\section{BACKGROUND}

Breast cancer refers to the formation of a malignant tumour in the breast tissue. In the year 2019, there was an estimated 268,600 new cases of invasive breast cancer and 41,760 breast cancer related deaths in the United States.[1] There exists two forms of in situ breast cancer, or cancer that is confined to the site that it has originated, these are the ductal carcinoma in situ and lobular carcinoma in situ. ${ }^{[2]}$ Most cases of breast cancer, about $80 \%$, are invasive or have spread beyond the area they have originated. There are up to 21 different types of invasive breast cancer, all of which vary in terms of cell morphology, growth, and architecture patterns. ${ }^{[3]}$ According to the American Cancer Society, any given woman living in the United States has a $12.4 \%$, or about one in eight, chance of being diagnosed with breast cancer at some point during her life. Incidence rates of breast cancer increase with age in women until their 60s in which the rates begin to decrease, possibly due to lower rates of screening and/or incomplete detection in older women. ${ }^{4]}$

There are several studies going on in the present scenario on breast carcinoma. The need for this is very obvious. Breast cancer being the most common cancer among women and also responsible for most of the cancer related deaths in women. The need to diagnose breast carcinoma with confidence and at an early stage cannot be underestimated.

Breast cancer survival is strongly determined based on the stage of the cancer after diagnosis, with the 5-year relative survival rate being $99 \%$ for when the cancer is localized in one area, $85 \%$ for a regional spread of cancer, and $27 \%$ for distant-stage cancer.[5] The five year survival rates for early breast carcinoma is $90 \%$; which means that if breast carcinoma is diagnosed at an early stage, and the correct treatment instituted, ninety percent of these women will be able to survive for the next five years. Therefore, we need to develop a system that not only helps to diagnose breast carcinoma at an early stage but also diagnoses it with utmost accuracy, so that the correct treatment could be instituted with utmost confidence.

The complaint of breast lump creates anxiety in patients with most of them having concern that the lump is breast carcinoma; while many of breast lumps are benign. This makes it even more important to diagnose any breast lump with utmost accuracy, so as to alleviate the anxiety of the patient and ensure that the patients are not over treated.

The American Cancer Society recorded that between the years 1975 and 1989, breast cancer deaths steady climbed about $0.4 \%$ per year, but since then have dropped to 2015 for a total decrease in 39\%. This decrease has been attributed to early detection and management of breast cancer.[6] Therefore the usefulness of proper diagnosis of breast cancer at early stage cannot be underestimated .The most common methods for breast cancer screening including mammography, magnetic resonance imaging (MRI), breast ultrasound, clinical breast examination (CBE), and breast selfexams (BSE). Mammography is generally carried out for women who are aged for greater than 35 years; because young women have denser breasts and to decipher presence of malignant lump in such a case will be difficult. Similarly, ultrasonography of the breast will be an option for women less than 35 years with denser breast and also for pregnant ladies.

Benign lumps can be distinguished on ultrasonography with some typical characteristics like- well defined margin, oval or horizontal orientation, no vascularity and no spiculations. MRI is used as a screening tool for breast carcinoma in patients of high risk such as those with BRCA mutations. Whenever there are radiological features of concern, a USG guided biopsy of the lesion can be performed which will confirm the diagnosis in most instances. However, there can be discordance of results of radiological assessment and pathological assessment and therefore the use of triple assessment yield importance. Triple assessment combines clinical examination with FNAC/core cut biopsy and radiological assessment (using USG/mammography) to arrive at final diagnosis and yields a positive predictive value of $99.9 \%$ in most instances. In this study, we are aiming to test the accuracy of triple assessment.

\section{METHODS}

\section{Study Site}

The study was carried out on patients attending OPD as well as admitted in surgical indoor of Nalanda Medical College \& Hospital, Patna with the complains related to breast. They were subjected to triple assessment comprising of clinical examination, Radiological evaluation (mammography or USG) and Pathological evaluation (FNAC or Tru-Cut biopsy) in order to diagnose carcinoma of breast.

\section{Study Design \& Sample Size}

After obtaining clearance from the institutional ethics committee, this cross-sectional study was conducted from December 2017 to November 2019. All patients that presented to the OPD with breast lump, excluding those with an abscess or ulcer, were included in this study; which made a total of 50 patients.

\section{Data Collection}

After a thorough clinical examination and proper history taking, the patients were subjected to radiological evaluation (USG or Mammography) and FNAC.

\section{Radiological Evaluation}

i. USG- Breast ultrasonography was performed using a high frequency transducer of 7-12 MHZ. Ultrasonography is the preferred modality in women younger than thirty-five years of age; and denser breasts. There are some typical characteristics that suggest a lump to be benign, such as well-defined margin, horizontal orientation and no vascularity while those that are malignant have characteristics such as spiculations, taller than wide, microcalcifications etc. Also, ultrasonography aids in biopsy of the lesion and ensures more accurate yield.

Method- The patient was placed in a supine or oblique position, with ipsilateral arm above the head. The breast 
was scanned in either a transverse or sagittal or radial and antiradial planes. The retroareolar area was evaluated by angling the transducer in multiple planes to avoid the shadowy artefact produced by the nipple.

ii. Mammography- All the patients with any breast lesion were subjected to mammographic examination of the both breast. Even if any breast lesion was known to be malignant by clinical examination, the contralateral breast was subjected to mammographic examination because of the multicentric nature of lobular carcinoma of breast. Mammography is an option to analyse breast lesions for women of age greater than 35 years and can also be used to judge if the lesion is multifocal or multicentric so that proper treatment could be planned.

Method- The breast was compressed in between Plexiglas plates to lessen the thickness of the tissue through which the radiation passed. Two view of each breast were obtained. The mediolateral oblique (MLO) and the craniocaudal (CC). The MLO view gave the images of the greatest volume of breast tissue, including the upper outer quadrant and the axillary tail of Spence. Compared with the MLO view the CC view provided better visualization of the medial aspect of the breast and permitted greater breast compression. The dose of the radiation used was $0.1 \mathrm{C}$ Gy. The classical features of carcinoma breast on mammography were noted as Mass effect, Architectural distortion, Asymmetric density, Micro calcification and Skin thickening. On the basis of these features the breast lesion were grouped as benign, intermediate and malignant. Intermediate group included probably benign or probably malignant.

\section{Pathological Evaluation}

Fine Needle Aspiration Cytology- After clinical examination and radiological evaluation each patient was subjected to fine needle aspiration of the breast lump.

Method- The patient was asked to sit on a stool with affected breast fully exposed. After proper antiseptic cleaning the lump was fixed between left index finger and thumb. No anaesthesia was needed for this procedure. The syringe fitted with 20-gauge needle was held in the right hand and the needle was introduced into the lump while constant suction was maintained in different direction inside the lump. Before withdrawal of the needle the syringe was disconnected to avoid suction of cellular material and tissue fluid into the syringe. After withdrawal of needle, firm pressure was maintained for 2 minutes at the site of puncture to avoid bleeding and haematoma formation. Then pressure dressing was applied for $24 \mathrm{hrs}$. After proper fixation and staining the slides were examined under light microscope for cytological diagnosis. On the basis of cellular details, majority of smears were diagnosed as benign or malignant but in few instances no diagnosis could be made due to inadequacy or absence of cells, while in others the diagnosis of malignancy was not certain. In this study the equivocal case were grouped under malignant.
Finally, all the patients were subjected to appropriate surgical intervention and the specimen obtained was send for histopathological examination for definitive diagnosis. Its result was compared with the combined results of clinical examination, radiological examination and pathological examination so called triple assessment.

\section{Statistical Methods}

The results of each modality were compared with histopathology report, to determine the sensitivity, specificity, positive predictive value and negative predictive value for each modality when they were used alone and when they were used in combination as part of triple assessment. Analysis was done using the chi-square test and $p$ value of $<0.05$ was considered to be significant.

\section{RESULTS}

The observation of this series is based on clinical examination, Radiological diagnosis and cytopathological study of 50 cases, having any symptom related to breast, who were admitted in the department of surgery, Nalanda Medical College \& Hospital, Patna. The main symptom related to breast was lump, which was of diverse aetiological nature and patients were of different age groups.

\begin{tabular}{|ccc|}
\hline Lumps & No. of Cases & Percentage \\
Malignant & 20 & 40 \\
Benign & 30 & 60 \\
Total & 50 & 100 \\
\hline Table 1. Comparative Incidence of Malignant and Non-Malignant \\
Lumps. It Shows That 40 \% of Total Cases were Malignant and Rest \\
were Benign with a Total of $60 \%$.
\end{tabular}

\begin{tabular}{|ccc|}
\hline Age Group in Years & No. of Cases & Percentage \\
$20-30$ & 11 & 22 \\
$30-40$ & 15 & 30 \\
$40-50$ & 08 & 16 \\
$50-60$ & 9 & 18 \\
$>60$ & 7 & 14 \\
\hline Table 2. Number of Patients in Each Age Group \\
\hline
\end{tabular}

Most of the patients were of 30-40 yrs. followed by those between 20-30 yrs.; constituting $30 \%$ and $22 \%$ respectively.

Lump in breast was more common on the right side $(56 \%)$ as compared to the left (42\%). Bilateral involvement of breast is very rare i.e. (02\%). Upper outer quadrant was the commonest site of origin (58\%) of lump breast. Next followed by upper inner (18\%), lower outer (12\%) and then lower inner $(4 \%)$. In the central part of breast below the nipple and areola only $8 \%$ was present.

Histopathological examination confirmed 20 patients to be malignant and 30 patients to be benign. The most common condition among the benign lump was fibroadenoma (50\%). The type of carcinoma present was ductal carcinoma in all the malignant lumps detected.

\begin{tabular}{|ccccc|}
\hline & Nature of Lump & $\begin{array}{c}\text { Histopathological } \\
\text { Examination } \\
\text { Malignant }\end{array}$ & Benign \\
Triple assessment & Malignant & 20 & 1 & 21 \\
& Benign & 0 & 29 & 29 \\
& Total & $\mathbf{2 0}$ & $\mathbf{3 0}$ & $\mathbf{5 0}$ \\
\hline \multirow{2}{*}{ Table 3. Results of Triple Tests Combining all Three Modalities and } \\
\\
Histopathology Confirmation \\
\hline
\end{tabular}


- True Positives-20

- True Negatives-29

- False Positives-1

- $\quad$ False Negatives-0

- Positive Predictive Value-95.23\%

- $\quad$ Negative Predictive Value- $100 \%$

- Sensitivity-100\%

- $\quad$ Specificity- $96.66 \%$

- Accuracy-98\%

- Error-2\%

\begin{tabular}{|cccccc|}
\hline & Sensitivity & Specificity & NPV & PPV & Accuracy \\
CBE & 90 & 86.66 & 92.8 & 81.8 & 88 \\
Radiological & 90 & 96.66 & 93.5 & 94.73 & 94 \\
Assessment & 95 & 96.66 & 96.66 & 95 & 96 \\
FNAC & 100 & 96.66 & 100 & 95.23 & 98 \\
Triple test & Table 4. Analysis of Triple Tests and its Components \\
\hline \multicolumn{4}{r}{}
\end{tabular}

\section{DISCUSSION}

The leading cause of cancer related deaths worldwide in females is breast cancer. The means of improving the survival rate is only early diagnosis. Therefore, various studies have been performed to judge the most appropriate tests that can yield accurate results, so that correct treatment could be instituted, and overtreatment be avoided. Clinical examination alone is not sufficient to decide on the course of treatment because of its high error of $12 \%$ as found in this study. Clinical examination alone can never be relied upon as an investigation modality to come to come to a definitive diagnosis. This is because clinical examination can diagnose breast carcinoma with certainty only when the carcinoma presents at an advanced stage. Furthermore, clinical examination is not a reproducible thing. It is rather a subjective thing and may vary from person to person. Therefore, clinical examination as a sole modality can never be accepted as a means of diagnosing breast carcinoma. These facts are well supported by our study too, as it had an accuracy of only $88 \%$.FNAC and mammography had similar specificity of $96.6 \%$, but sensitivity of FNAC (95\%) was higher than mammography $(90 \%)$.

Among FNAC and mammography or ultrasonography, both have some merits and demerits. Also, the yield of FNAC and similarly its accuracy can be increased if the biopsy was aided by ultrasonography which is USG guided biopsy.

From these observations it is clear that no single modality can be relied upon as an individual test and the highest level of accuracy can very well be obtained when all the three modalities are used in combination to arrive at a diagnosis which is in the form of triple assessment. The combined use of these three modalities, as part of the triple assessment yielded a sensitivity and negative predictive value of $100 \%$; thus, proving that it can be relied upon as gold standard modality for diagnosing breast carcinoma and definitive treatment be instituted based on its results.

Similar results were also obtained in other studies. Jane M et al, 2010 yielded a sensitivity and negative predictive value of $100 \%$ and specificity of 99.3\%.[7] Chalaya P et al, 2013 and Mehta et al, 2017 yielded a sensitivity, specificity, negative predictive value and positive predictive value of $100 \%$ each,[8.9] for triple assessment in diagnosing carcinoma breast.

\section{CONCLUSIONS}

With a sensitivity and negative predictive value of 100, triple assessment can very well be relied upon to diagnose breast lumps with confidence and institute definitive treatment. The study has proved that using a combination of three modalities is the best way to diagnose a breast lump correctly.

\section{REFERENCES}

[1] American Cancer Society. Breast Cancer Facts and Figures 2019-2020

https://www.cancer.org/content/dam/cancer-

org/research/cancer-facts-andstatistics/breast-cancerfacts-and-figures/breast-cancer-facts-and-figures-20192020.pdf.

[2] Allred DC. Ductal carcinoma in situ: terminology, classification and natural history. J Natl Cancer Inst Monogr 2010;2010 (41):134-8.

[3] Dieci MV, Orvieto E, Dominici M, et al. Rare breast cancer subtypes: histological, molecular and clinical peculiarities. Oncologist 2014;19 (8):805-13.

[4] Ravdin PM, Cronin KA, Howlader N, et al. The decrease in breast cancer incidence in 2003 in the United States. N Engl J Med 2007;356 (16):1670-4.

[5] Howlader N, Noone AM, Krapcho M, et al. SEER Cancer Statistics Review, 1975-2014, Bethesda, MD: National Cancer Institute, April 2017.

[6] Berry DA, Cronin KA, Plevritis SK, et al. Effect of screening and adjuvant therapy on mortality from breast cancer. N Engl J Med 2005;353 (17):1784-92.

[7] Jan M, Mattoo JA, Salroo NA, et al. Triple assessment in the diagnosis of breast cancer in Kashmir. Indian J Surg 2010;72 (2):97-103.

[8] Chalaya PL, Lema MK, Mabula JB, et al. Triple assessment as a preoperative diagnostic tool for breast cancer at Bugando Medical Centre in Northwestern Tanzania. Tanzan J Health Res 2013;15 (4):223-9.

[9] Mehta R, Tewari K, Goyal N, et al. Triple approach for diagnosing breast lesions - experience at a tertiary care hospital. J Mar Med Soc 2017;19 (2):123-7. 